\title{
Early nucleation stages of low density InAs quantum dots nucleation on GaAs by MOVPE
}

G. Torelly ${ }^{1,2}$, R. Jakomin ${ }^{1,3}$, L. D. Pinto ${ }^{1,2}$, M. P. Pires ${ }^{1,4}$, J. Ruiz ${ }^{1,2}$, P. G. Caldas ${ }^{5}$, R. Prioli ${ }^{5}$, H. Xie ${ }^{6}$, F. A. Ponce $^{6}$ and P. L. Souza ${ }^{1,2}$

${ }^{1}$ Instituto Nacional de Ciência e Tecnologia de Nanodispositivos Semicondutores - DISSE - Brazil

${ }^{2}$ Laboratório de Semicondutores, Pontifícia Universidade Católica do Rio de Janeiro, Rua Marquês de São Vicente 225, Rio de Janeiro, 22451-900, Brazil

${ }^{3}$ Campus de Xerém, Universidade Federal do Rio de Janeiro, Duque de Caxias - RJ, Brazil

${ }^{4}$ Instituto de Física, Universidade Federal do Rio de Janeiro, Rio de Janeiro- RJ, Brazil

${ }^{5}$ Departamento de Física, Pontifícia Universidade Católica do Rio de Janeiro, Rua Marquês de São Vicente 225, Rio de Janeiro, 22451-900, Brazil

${ }^{6}$ Department of Physics, Arizona State University, Tempe, Arizona 85287-1504, USA

e-mail corresponding author: plustoza@cetuc.puc-rio.br

\begin{abstract}
An investigation of ultra-thin InAs layers deposited on GaAs is carried out combining theoretical calculations with results of high-resolution transmission electron microscopy, atomic force microscopy and photoluminescence. Five period InAs/GaAs epilayers were grown by metalorganic vapor phase epitaxy at a very low growth rate, with different InAs deposition times, in order to investigate the morphological and optical evolution from extended 2D InAs flat areas of various thicknesses, starting at 1 monolayer, to the nucleation of 3D InAs islands. The coexistence of extended monolayer-flat 2D terraces of different thicknesses and 3D islands is demonstrated. Optically active InAs 2D terraces with a thickness beyond the critical value are detected. For longer deposition times, quantum dots are nucleated and their size increases at the expense of the 3 monolayer thick 2D layers.
\end{abstract}

Keywords: A.1 nanostructures, A.3 metalorganic vapor phase epitaxy, B.1 arsenates, B.2, semiconducting III-V materials 


\section{Introduction}

The production of quantum dots (QDs) by the Stranski-Krastanov technique has been established in the 1990s as the main approach to produce these nanostructures for device applications due to the relative simplicity of the method, low cost and scalability [1-5], as compared to top-down approaches. In the Stranski-Krastanov method a material is deposited with a different lattice parameter from that of the substrate, leading to a transition from a 2D to a 3D growth mode. Since then, there have been several reports on the early stages of the nucleation of InAs QDs on GaAs [6-8], motivated, in particular, by the potential od such QD structures for the fabrication of lasers in the telecommunications wavelength range with improved performance and lower costs [9]. The formation and morphological evolution of InAs QDs grown on GaAs substrates by molecular beam epitaxy (MBE) [6], metalorganic vapor phase epitaxy (MOVPE) [7] and chemical beam epitaxy (CBE) [8] have been investigated and the transition from 2D to 3D growth mode has been highlighted for the three epitaxial growth methods. Regardless of the growth technique employed (MBE, MOVPE or CBE), photoluminescence (PL) spectra of $\ln A s / G a A s$ structures presented two different kinds of emissions depending on the quantity of the material used for the growth [6-8]. A narrow peak around $1.4 \mathrm{eV}$ due to the $2 \mathrm{D}$ InAs layer formed prior to the onset of QD nucleation was observed when the thickness of the InAs layer was below the critical value. The exact energy depended on the InAs 2D layer growth conditions and thickness. After further growth, a broader peak was observed at a lower energy (between 1.00 and $1.35 \mathrm{eV}$ ), which was 
attributed to the QD formation. Depending on the growth and measurement conditions, both peaks could be simultaneously observed.

Earlier, in the 1980s, strained ultra-thin InAs layers were obtained on GaAs substrates forming quantum wells (QWs) of various thicknesses, ranging from below one monolayer (ML) to about $4 \mathrm{MLs}$, exhibiting strong PL emission with full width at half maximum (FWHM) between 4 and $20 \mathrm{meV}$ [10-15]. Only one PL peak was observed near the bandedge energy of the InAs QW, attributed to a specific QW thickness. No PL splitting, meaning two peaks emitted by extended flat areas of different thicknesses on the same QW layer, was observed. This PL splitting has been previously reported for ultrathin QWs grown lattice matched to the substrate (GaAs/AIGaAs [16], InGaAs/InP [17]). More recently, possible mechanisms for the nucleation of InAs QDs on GaAs by MOVPE have been thoroughly described [18] and improvements in the QDs size distribution and morphology by playing with different growth parameters have been reported [19-23]. However, there still remain challenges for a full understanding of mechanisms of QD formation. Even more so as reports on QD structures grown by MBE have dominated the literature, while relatively little has been published on QDs deposited by MOVPE.

A detailed investigation of a series of samples where InAs is deposited by MOVPE on GaAs, at a very low growth rate and for different lengths of time, is reported. Combining the results of ex-situ characterization techniques such as atomic force microscopy (AFM), high resolution transmission electron microscopy (HRTEM) and in-situ reflectance anisotropy spectroscopy (RAS) with PL, it was possible to probe the early stages of the QD nucleation, verify the existence of different 2D extended flat domains, detect PL splitting, obtain PL 
emission from a 2D domain with thickness above the critical value and unambiguously demonstrate the material consumption from the 2D layers to nurture the 3D islands formed.

\section{Experimental details}

On a semi-insulating exactly oriented $(100) \pm 0.5^{\circ} \mathrm{GaAs}$ substrate with hydrogen as a carrier gas and trimethylgalium (TMGa) as a precursor, a $400 \mathrm{~nm}$ GaAs buffer layer is grown by MOVPE in a horizontal AIX200 reactor at $100 \mathrm{mbar}$ and $630^{\circ} \mathrm{C}$ with a V/III ratio equal to 23 and a growth rate of $0.38 \mathrm{~nm} / \mathrm{s}$, determined by TEM images (fig.1). The mentioned temperatures are the thermocouple ones. The GaAs buffer layer was observed to be smooth, presenting surface steps with approximately $0.28 \mathrm{~nm}$ in height and $\sim 1.8$ arc minutes off the [001] direction. Following the GaAs growth, the temperature is reduced to $490^{\circ} \mathrm{C}$, which takes approximately 4 minutes. Once this temperature is reached, 2 minutes are allowed for temperature stabilization before the arsine $\left(\mathrm{AsH}_{3}\right)$ flux is decreased by a factor of 4 , over an interval of $30 \mathrm{~s}$. At this point, the trimethylindium (TMIn) flux is switched from vent to the growth chamber and InAs is deposited for $t$ seconds with a V/III ratio equal to 32 . The zoom in fig. 1 shows a line scan where each peak represents a GaAs ML and the weak central peak, surrounded by two minima, reveal a $2 \mathrm{ML}$ thick InAs layer. The deposition times used were $3.6 \mathrm{~s}, 4.8 \mathrm{~s}, 6.0 \mathrm{~s}, 7.2 \mathrm{~s}, 8.4 \mathrm{~s}, 9.6 \mathrm{~s}, 10.8$ $\mathrm{s}$ and $12.0 \mathrm{~s}$. Once the growth is halted, the sample is then annealed at the same temperature $\left(490^{\circ} \mathrm{C}\right.$ ) for $12.0 \mathrm{~s}$. Afterwards, the temperature is raised back to $630^{\circ} \mathrm{C}$ and the arsine flux is increased to its original value. As the temperature is raised, GaAs is deposited for 15 seconds. In the case InAs QDs are nucleated, this GaAs barrier layer is, in fact, the QDs capping layer which can either completely cover them, if their height is less than about $5 \mathrm{~nm}$, or limit their height to 
this layer thickness (slightly over $5 \mathrm{~nm}$ ). Once the temperature reaches $630^{\circ} \mathrm{C}, 1.5$ minutes are allowed for temperature stabilization before a $16 \mathrm{~nm}$ thick GaAs layer is deposited. This is the so-called In flush method [24-26]. Samples were grown with either a single or a five periods InAs/GaAs layers. The one and five period samples will be identified, throughout the text, by the number of periods times the deposition time of the InAs layers. As an example, sample $1 \times 3.6$ is the one period InAs/GaAs sample for which the InAs layer was deposited for $3.6 \mathrm{~s}$, while the $5 \times 3.6$ is the five period InAs/GaAs sample for which the InAs layer was deposited for $3.6 \mathrm{~s}$. The same nomenclature is applied to all samples.

The PL measurements have been carried out with the $514 \mathrm{~nm}$ line of an $\mathrm{Ar}^{+}$laser for optical excitation. The signal was dispersed by a $250 \mathrm{~mm}$ monochromator and detected by a nitrogen cooled Ge photodetector. Measurements were performed at $16 \mathrm{~K}$. AFM using tapping mode was employed to obtain images and profiles of the surface of the samples. Additionally, for the samples in which QDs were nucleated, it was possible to determine the presence, height and density of QDs on InAs layers with free standing QDs. High resolution transmission electron microscopy images in cross section were used to determine the layers' thicknesses and QD height, as well as to observe the changes in QD density in the buried layers. Cross-section TEM thin foils were prepared by mechanical wedge polishing followed by argon-ion milling using a 2 kV accelerating voltage at liquid $\mathrm{N}_{2}$ temperatures. The InAs QD layers were studied with Philips CM-200 transmission electron microscope, operating with an accelerating voltage of $200 \mathrm{kV}$.

\section{Results and Discussion}

Morphological aspects 
AFM images were made for the samples having five layers of InAs grown for different lengths of time. As observed in fig. 2 (a), QD formation is detected for single period samples with $10.8 \mathrm{~s}$ InAs deposition time, whereas QDs are visible on the surfaces of the five periods samples already with $8.4 \mathrm{~s}$ InAs growth time. The fact that for the five period samples the 2D to 3D growth mode transition is triggered for a thinner layer is likely to be caused by the accumulated strain from the buried InAs layers [27].

In order to build QD size distribution histograms, the AFM images for samples $1 \times 10.8$, $5 \times 10.8,1 \times 12.0$ and $5 \times 12.0$ were analyzed using an optimized procedure recently developed to accurately determine both the height and QD density [28]. Fig. 3 shows the obtained histograms. Samples grown for $8.4 \mathrm{~s}$ and $9.6 \mathrm{~s}$ presented QDs only when five InAs layers were deposited. The histograms have been fitted by a log-normal function [29]. The density of QDs for the sample grown for $10.8 \mathrm{~s}$ containing a single InAs layer is $3.810^{8} \mathrm{~cm}^{-2}$ (see fig. 3 (a)). An equivalent one period sample grown for $9.6 \mathrm{~s}$ does not have QDs, showing that the nucleation of QDs sets in rather abruptly. This behavior is expected since the QD nucleation is known to have an ultrafast initial evolution once the critical thickness of the InAs layer is reached [6]. The maximum of the QDs fitted histogram for the one period sample grown for $10.8 \mathrm{~s}$ occurs for a QD height equal to $7.7 \mathrm{~nm}$. For the sample with five periods, the QD density on the surface increases by a factor of about 3, while the QD height increases approximately $25 \%$. As shown in figs. 3 (c) and (d), for a growth time of $12.0 \mathrm{~s}$ the QD density and height remain essentially the same whether one layer or 5 periods of InAs are deposited.

Fig. 4 shows TEM images for samples $5 \times 8.4,5 \times 9.6$ and $5 \times 12.0$, the samples being tilted to excite the 002 spot in the diffraction pattern. Images for samples $5 \times 8.4$ and $5 \times 9.6$ show that no 
QDs are nucleated in the first layer, which is consistent with AFM images obtained for the samples with a single InAs layer. The images also show that the density of dots in each layer increases sequentially up to the third layer, after which the dot density per layer remains constant. In the case of sample $5 \times 12.0$, QDs appear already in the first layer and their density is not influenced by the number of InAs layers, confirming the results from the AFM histograms discussed before (fig. 3). The HREM image shown in fig. 1 reveals a thickness for the buried InAs wetting layer of about $2 \mathrm{ML}$, in agreement with previous reports of InAs QDs nucleated on GaAs surfaces [6-8].

The transition from a $2 \mathrm{D}$ to a 3D growth mode can also be probed by in-situ RAS spectra which provide information on the surface reconstruction occurring during the MOVPE growth. Fig. 5 shows RAS spectra for the GaAs surface before the InAs deposition and for the InAs layers grown for different lengths of time. The negative minimum at $2.6 \mathrm{eV}$ is characteristic of a $\mathrm{c}(4 \mathrm{x}$ 4) surface reconstruction, typical of GaAs. The surface reconstruction for $\ln A s$ is $c(2 \times 4)$ and it is characterized by a positive signal at a slightly lower energy [30,31]. The relaxed InAs should have a positive peak between 2.3 and $2.4 \mathrm{eV}$. However, the RAS peak for strained InAs should be at a slightly higher energy, as it is observed in fig. 5 . The $c(2 \times 4)$ signal increases with InAs growth time, indicating a larger InAs surface coverage. For samples $5 \times 6.0$ and $5 \times 7.2$, a maximum signal is reached and the surface presents a predominantly $c(2 \times 4)$ reconstruction. For sample 5x8.4, QDs are nucleated and the RAS signal suffers a slight decrease at $2.6 \mathrm{eV}$. Moreover, a strong RAS signal at $4.0 \mathrm{eV}$ indicates a 3D growth mode. As shown in fig. 5, the RAS signal reaches a maximum at $4.0 \mathrm{eV}$ when the QD nucleation sets in (5x8.4 sample), consistent with the AFM image of fig. 2 (a). A high signal is also observed for sample $5 \times 3.6$, which 
decreases with growth time up to 6.0 s. For these growth times no QDs are observed, but on the other hand, at such short deposition times, the surface is quite rough, smoothening out as the growth time increases to 6.0 or $7.2 \mathrm{~s}$. The high energy RAS signal reaches a minimum for sample $5 \times 6.0$, revealing a $2 \mathrm{D}$ growth mode. For sample $5 \times 7.2$, it is possible that the surface suffers changes since the InAs layer is in a metastable state, at the verge of nucleating QDs. The further increase in the $4.0 \mathrm{eV}$ signal when QDs are nucleated (sample 5x8.4) indicates an increase in anisotropy attributed to the elongation of the QDs [30].

\section{Optical aspects}

Fig. 6 shows the PL spectra measured at $16 \mathrm{~K}$ in the range between 1.38 and $1.52 \mathrm{eV}$ of the samples with five periods of InAs grown for different lengths of time. The striking feature of these PL spectra is that they show PL peaks (FWHM between 6 and $18 \mathrm{meV}$ ) at four specific energies, namely: $1.494 \mathrm{eV}, 1.475 \mathrm{eV}, 1.450 \mathrm{eV}$ and $1.410 \mathrm{eV}$, which correspond to the 2D InAs strained layers of different thicknesses. The FWHMs indicate that the buried interfaces have excellent quality [32] and that free exciton emissions are observed [17].

Fig. 7 shows the lower optical transition energy at $16 \mathrm{~K}$ as a function of QW width, obtained by solving the Schrödinger equation using the effective mass model taking strain into account. The calculations assume a $70 \%$ band offset for the conduction band, $30 \%$ for the valence band and an energy gap for the strained InAs equal to $0.784 \mathrm{eV}$ [33]. Additionally, a 4.35 meV exciton binding energy is included based on an extrapolation for InAs of available experimental data for GaAs and InGaAs alloys [33, 34], as well as on theoretical predictions [35]. The PL experimental peak positions are represented by different symbols, while the calculated values are depicted 
by a vertical dash. The line is just a guide for the eyes. According to the calculations, the observed PL peak at $1.494 \mathrm{eV}$ for sample $5 \times 3.6$ corresponds to emission from a 3.6 angstroms thick QW. This thickness is equivalent to about $1 \ln A s M L$, considering that one strained $\ln A s$ monolayer is 3.28 angstroms thick, and corresponding to an InAs growth rate of $\sim 0.3 \mathrm{ML} / \mathrm{s}$. The estimated growth rate leads to InAs layer thickness consistent with TEM measurements for the first InAs layers prior to QDs nucleation. 2D InAs extended monolayer terraces are formed which have an area larger than the Bohr radius (around 40 angstroms), as it should be when a single narrow PL peak is observed. Such extended monolayer flat areas have already been reported for the GaAs/AlGaAs system [16] and for InGaAs layers lattice matched to InP [17]. It should be noted that the layers probed by PL are buried. The high surface recombination typically does not allow observation of PL originating in the top GaAs layer. As pointed out before and observed in fig.1 and fig. 4, the InAs surface is significantly smoothened out following the annealing procedure. This result is consistent with the narrow FWHM, $7 \mathrm{meV}$, observed for such peak.

For samples $5 \times 4.8$ and $5 \times 6.0$, the $1 \mathrm{ML}$ peak is not present and a narrow peak around 1.475 $\mathrm{eV}$ is observed. According to the calculations shown in fig. 7, such peak corresponds to a 5.3 angstroms (1.6 MLs) thick InAs QW. PL emission from a non-integer monolayer has already been reported for different materials and growth techniques $[8,10,11]$. Assuming the same growth rate, the thickness of the layers for these two samples should be 1.4 and 1.8 MLs, very close to the calculated 1.6 MLs. The non-integer monolayer thick InAs, evidenced by a single PL emission, can be interpreted as a result of the recombination of excitons which experience a local potential in average equivalent to that of a $1.6 \mathrm{ML}$ thick InAs layer, meaning that there are 
regions that are $1 \mathrm{ML}$ thick and others $2 \mathrm{ML}$ thick which have a lateral dimension smaller than the Bohr radius. Although the microroughness has been essentially eliminated by annealing, roughness on a nanometer scale is still detected by $\mathrm{PL}$ for these two samples. It is relevant to note that for both samples the same specific average layer thickness is observed. As the growth time of the InAs increases to $7.2 \mathrm{~s}$ and $8.4 \mathrm{~s}$, two PL peaks are simultaneously observed, one at $1.450 \mathrm{eV}$ and the other at $1.410 \mathrm{eV}$, which are assigned to a 2 and $3 \mathrm{ML}$ thick InAs QWs, respectively, fully matching the calculations shown in fig. 7. We conclude that for 7.2 and 8.4 seconds growth time, the samples have two domains of 2D InAs extended flat terraces: one $2 \mathrm{ML}$ thick and another $3 \mathrm{ML}$ thick. It is interesting to note that a $3 \mathrm{ML}$ thick layer should, in principle, not be observed because it is thicker than the threshold thickness for QD formation. PL of InAs QWs thicker than the critical thickness has, nevertheless, already been reported [10, 11, 13]. Non-integer monolayer thick InAs is not observed for longer growth times because the roughness on the nanometer scale is reduced. The remaining roughness has a larger lateral extent, implying that the excitons are submitted to the potential of an integer ML thick layer. For the samples with an InAs layer grown for even longer times, such as the $5 \times 9.6,5 \times 10.8$ and $5 \times 12.0$ ones, the highest energy PL peak observed is at $1.45 \mathrm{eV}$, which is assigned to a $2 \mathrm{ML}$ thick InAs QW. It is surprising to note that the $3 \mathrm{ML}$ peak is absent for the samples for which InAs was deposited for even longer times than $8.4 \mathrm{~s}$, even though the three of them have a clear emission from a 2 ML thick InAs QW. According to figs. 2 (b), (c) and (d), mature QDs with a density above $1 \times 10^{9} \mathrm{~cm}^{-2}$ are present for these samples. The explanation for the absence of the $3 \mathrm{ML}$ QW PL peak is that with longer growth time, the QDs have time to increase in size collecting In atoms from the unfinished $3 \mathrm{ML}$ thick terraces around, leaving the wetting layer 
with a $2 \mathrm{ML}$ thickness (fig. 1), which is, in fact, about the critical thickness for QD formation in the Stranski-Krastanov method.

An additional weak emission around $1.497 \mathrm{eV}$ is detected for sample $5 \times 12.0$, as well. In fact, if the scale in the PL intensity axis (fig.6) is expanded for the energy range between 1.45 and $1.52 \mathrm{eV}$, one observes that two very weak emissions are present for all samples except for the 5x3.6: one at $1.521 \mathrm{eV}$ due to the GaAs buffer and barrier layers and another one at 1.495, which is also present in the buffer layer (PL of a sample with just a GaAs buffer layer grown under the same growth conditions and on the same type of substrate as that of the investigated samples was measured), attributed to an impurity related transition, most likely from conduction band to C acceptor. PL from GaAs is very weak compared to the lower energy peaks because the carriers most likely are trapped by the lower energy InAs QWs and QDs. It is possible to observe the very weak GaAs related PL emission in the expanded PL spectrum of sample 5x3.6, as well. However, the second weak emission at $1.495 \mathrm{eV}$ is not detected. It is possible that such emission exists but is involved in the much stronger (about two orders of magnitude) 1 InAs ML thick peak at $1.494 \mathrm{eV}$. It should be pointed out that the latter peak and the impurity related one at $1.495 \mathrm{eV}$, observed for the other samples, are very different in nature. The power dependence of the intensity for the conduction band to acceptor level transition is linear, whereas for the 1 InAs ML thick QW, the dependence is superlinear versus the excitation power for the range used. Moreover, the FWHM for the latter is only $7 \mathrm{meV}$ while for the impurity peak in all investigated samples is approximately 10 meV.

Fig. 8 shows the PL spectra at $16 \mathrm{~K}$ in the range between 1.05 and $1.55 \mathrm{eV}$ for the samples with InAs grown for a time equal to or longer than $8.4 \mathrm{~s}$, for which QDs are nucleated. The 
emissions between 1.10 and $1.35 \mathrm{eV}$ are attributed to QDs, the higher the energy, the smaller the dot. According to the AFM image shown in fig. 2 (a), a low density of small dots ( $1 \times 10^{8}$ $\mathrm{cm}^{2}$ ) is obtained for sample $5 \times 8.4$ and therefore a weak QD PL peak is detected at $1.3 \mathrm{eV}$. Larger dots are present for sample $5 \times 9.6$, as revealed by the QD PL peak at lower energy (around 1.15 eV) and confirmed by the AFM images (fig. 2 (b)). Still in fig. 2 (b), it is clear that there are QDs of very different sizes, giving rise to the shoulders present in the PL spectrum of this sample. The spectrum for sample $5 \times 10.8$ also shows a broad band centered around $1.3 \mathrm{eV}$ with two lower energy shoulders due to different QDs families, in accordance with the AFM images in fig. 2 (c). Finally, the PL spectrum for sample 5x12.0, which has the largest QD density present (fig. $2(\mathrm{~d})$ ), is dominated by the QD emission of small (PL peak energy at $1.3 \mathrm{eV}$ ) and large dots (PL peak energy at $1.1 \mathrm{eV})$.

Three dimensional calculations of the Schrödinger equation predict that the QD emission energy as a function of QD height at $16 \mathrm{~K}$ should follow the solid and the dashed lines for QD radius equal to $5 \mathrm{~nm}$ and $6 \mathrm{~nm}$, respectively, as depicted in fig. 9. A disk shaped QD is assumed for the calculations. The experimental points are represented by different symbols for samples $5 \times 8.4,5 \times 9.6,5 \times 10.8$ and $5 \times 12.0$, as described in the inset. According to fig. 9, all four samples should have QDs about $2 \mathrm{~nm}$ high. In the case of sample $5 \times 8.4$, the QDs are fully covered by the GaAs capping layer grown at low temperature $\left(490^{\circ} \mathrm{C}\right)$. Samples $5 \times 9.6$ and $5 \times 10.8$ show a broad QD emission, with two clear shoulders each, which can be fitted by three PL peaks. The two higher energy peaks at $1.3 \mathrm{eV}$ and $1.22 \mathrm{eV}$, are attributed to fully covered QDs with heights around $2 \mathrm{~nm}$ and $3 \mathrm{~nm}$, respectively, assuming they maintain a $5 \mathrm{~nm}$ radius. The third emission, at $1.15 \mathrm{eV}$ for sample $5 \times 9.6$, should come from the larger dots which are truncated by the In 
flush procedure, and limited to $5 \mathrm{~nm}$ height, the thickness of the capping layer. In fact, that is in full agreement with the calculations, as shown in fig. 9 by the pink triangle to the right. It is true that according to the AFM images and histograms, there are many QDs larger than $5 \mathrm{~nm}$, however these are surface QDs and not the buried ones, probed by PL. The shoulder at the lowest energy in the PL spectrum for sample 10.8 is at $1.08 \mathrm{eV}$. As the larger QDs have their height limited to $5 \mathrm{~nm}$, the lower PL peak energy, as compared to that of the $5 \times 9.6$ sample, can only be explained if the diameter of the QDs is larger. Indeed, if we observe the results of the simulations for a different QD diameter, shown by the dashed line in fig. 9, one concludes that the QD radius should be between 5 and $6 \mathrm{~nm}$. Sample $5 \times 12.0$ gives rise to two clear QD PL peaks, one at $1.3 \mathrm{eV}$, attributed to small QDs, fully covered by the capping layer and one at 1.07 eV, attributed to the larger buried dots. Such PL peak energy corresponds to that emitted by QDs $5 \mathrm{~nm}$ high, as long as the QD radius increases to about $5.5 \mathrm{~nm}$ (see hexagon in fig. 9). It is clear that the diameter of QDs nucleated for longer periods of time have a larger volume, as observed in the AFM and TEM images. It should be pointed out that the values of QDs dimensions and the peak energies of their PL emissions may not be exact given the experimental errors and theoretical assumptions, but the trends are, beyond any doubts, strictly followed.

It is important to be critical with respect to the excellent agreement of the calculated transition energies as a function of the thicknesses of the InAs QWs with the experimental data. Several published data agree very closely $[10,11,13,14]$ with our results. There is one [12] which shows lower transition energies for the thin 2D InAs QWs. The authors claim that higher energies are a consequence of intermixing. It should be argued whether in the presently 
investigated samples $\mathrm{In} / \mathrm{Ga}$ intermixing is significant, leading to the formation of an InGaAs alloyed QW. Little intermixing is expected for growth temperatures below $550^{\circ} \mathrm{C}$ [36]. Our samples were subjected to temperatures as high as $630^{\circ} \mathrm{C}$, for which a $\mathrm{Ga} / \mathrm{In}$ interdiffusion coefficient for strained layers equal to $1.39 \times 10^{-19} \mathrm{~cm}^{2} / \mathrm{s}$ is expected [36]. For the average time the sample was subjected to such temperatures, an interdiffusion length of at least 2 angstroms is expected. Even though this strain induced intermixing occurs in the investigated QWs, it should not be significant. Other sources of inaccuracy in the calculations of the PL peak energies are the choice of the band gap of the strained InAs layer, which is used as an input, and the estimation of the exciton binding energy. A value of $0.784 \mathrm{eV}$ for the band gap was used, following reference [37]. However, this value is controversial, lower values can also be found in the literature [38]. The exciton binding energy should vary with QW width [33, 34], but we have assumed a constant value for such thin layers since appropriate experimental data is not available for them.

\section{Conclusion}

In a series of eight five period samples of InAs grown by MOVPE on GaAs for deposition times between $3.6 \mathrm{~s}$ to $12.0 \mathrm{~s}$ the evolution of the surface from $2 \mathrm{D}$ extended monolayer flat terraces of different thicknesses to finally reach the 3D Stranski-Krastanov growth mode is observed. As the growth time increases from $3.6 \mathrm{~s}$ to $8.4 \mathrm{~s}, 2 \mathrm{D}$ extended flat areas with $1 \mathrm{ML}$ (3.6 s), $1.6 \mathrm{ML}(4.8 \mathrm{~s}$ and $6.0 \mathrm{~s}$ ), $2 \mathrm{MLs}(7.2 \mathrm{~s}$ and $8.4 \mathrm{~s})$ and $3 \mathrm{MLs}(7.2 \mathrm{~s}$ and $8.4 \mathrm{~s})$ were detected by PL of stacked layers. For a deposition time of $8.4 \mathrm{~s}$, in addition to the coexistence of extended flat areas of different thicknesses, QDs are nucleated, giving rise to a third PL 
emission. For longer deposition times, larger and a higher density of QDs are grown which consume the material of the $3 \mathrm{ML}$ thick terraces, as indicated by the absence of the corresponding PL peak for deposition times equal to or longer than 9.6 s. For these longer growth times more than one size family of QDs are formed, according to the PL spectra, but their heights are limited to the GaAs cap layer thickness of $5 \mathrm{~nm}$ due to the In flush capping method. Theoretical calculations of the PL emission energies as a function of QW thickness and QD height agree exceptionally well with the trend of the experimental data. The exact quantitative agreement should critically, be looked upon.

\section{Acknowledgements}

This work was partially supported by FAPERJ, CNPq, FINEP and CAPES. The research at ASU was supported in part by the National Science Foundation (NSF) and the Department of Energy (DOE) under NSF CA No. EEC-1041895, and in part by the NSF Materials World Network (DMR1108450).

\section{References}

[1] J. M. Gérard, in: Confined Electrons and Photons: New Physics and Applications, NATO ASI Series, Eds. C. Weisbuch and E. Burstein (Plenum, New York, 1994) 357.

[2] D. Leonard, M. Krishnamurthy, C. M. Reaves, S. P. Denbaars, P. M. Petroff, Direct formation of quantum-sized dots from uniform coherent islands of InGaAs on GaAs surfaces, Appl. Phys. Lett. 63 (1993) 3203.

[3] D. Bimberg, M. Grundmann, N. N. Ledentsov, Quantum Dot Heterostructures, John Wiley and Sons, Chichester, 1998, 22. 
[4] W. Seifert, N. Carlsson, J. Johansson, M.-E.Pistol, L. Samuelson, In situ growth of nanostructures by metal-organic vapour phase epitaxy, J. Crystal Growth 170 (1997) 39.

[5] P. M. Petroff, S. P. DenBaars, MBE and MOCVD growth and properties of self-assembling quantum dot arrays in III-V semiconductor structures, SuperlatticesMicrostruct. 15 (1994) 15.

[6] J. M. Gérard, O. Cabrol, J. Y. Marzin, N. Lebouché,J. M. Moison, Optical investigation of some statistic and kinetic aspects of the nucleation and growth of InAs islands on GaAs, Mater.Sci. Eng.B 37 (1996) 8.

[7] M. Geiger, A. Bauknecht, F. Adler, H. Schweizer, F. Scholz, Observation of the 2D-3D growth mode transition in the InAs/GaAs system, J.Crystal Growth 170 (1997) 558.

[8] J. Zribi, D. Morris, R. Arès, Formation and morphological evolution of InAs quantum dots grown by chemical beam epitaxy, J.Vac. Sci. Technol. B30 (2012) 051207.

[9] N. N. Ledentsov, M. Grundmann, F. Heinrichsdorff, D. Bimberg, V. M. Ustinov, A. E. Zhukov, M. V. Maximov, Zh. I. Alferov, J. A. Lott, IEEE J. Sel. Top. Quantum Electronics 6 (2000) 439.

[10] K. Taira, H. Kawai, I. Hase, K. Kaneko, N. Watanabe, Noninteger InAs monolayer well InAs/GaAs single quantum well structures grown by metalorganic chemical vapor deposition, Appl. Phys. Lett. 53 (1988) 495.

[11] J.M. Gérard, J. Y. Marzin, High quality ultrathin Inas/GaAs quantum wells grown by standard and low-temperature modulated-fluxes molecular beam epitaxy, Appl. Phys. Lett. 53 (1988) 568.

[12] M. Sato, Y. Horikoshi, InAs monomolecular plane in GaAs grown by flow-rate modulation epitaxy, J.Appl. Phys. 66 (1989) 851.

[13]M. Yano, K. Yoh, T. Iwawaki, Y. Iwai, M. Inoue, Structural stability of ultrathin InAs/GaAs quantum wells grown by migration enhanced epitaxy, J.Crystal Growth 111 (1991) 397.

[14] T. Noda, M. R. Fahy, T. Matsusue, B. A. Joyce, H. Sakaki, MBE growth and properties of monolayer and submonolayerInAs layer embedded in GaAs/AlAs quantum wells, J.Crystal Growth 127 (1993) 783.

[15] M. Ilg, M. I. Alonso, A. Lehmann, K. Ploog, M Hohenstein, Investigation of InAs submonolayer and monolayer structures of GaAs(100) and (311) substrates, J.Appl. Phys. 74 (1993) 7188. 
[16] E. S. Koteles, B. S. Elman, C. Jagannath, Y. J. Chen, Temperature-dependent optical spectra of single quantum wells fabricated using interrupted molecular beam epitaxial growth, Appl. Phys. Lett. 49 (1986) 1465.

[17] X. Liu, S. Nilsson, L. Samuelson, W. Seifert, P. L. Souza, Extended monolayer flat islands and exciton dynamics in Ga0.47 In 0.53 As/InP quantum-well structures, Phys. Rev. B 47 (1993) 2203.

[18] T. Chung, G. Walter and N. Holonyak Jr., Growth mwchanism of InAs quantum dots on GaAs by metalorganic chemical vapor deposition, Journal of Appl. Phys. 97 (2005) 053510.

[19] N. Nuntawong, S. Huang, Y. B. Jiang, C. P. Hains and D. L. Huffaker, Defect dissolution in strain-compensated stacked InAs/GaAs quantum dots grown by metalorganic chemical vapor deposition, Appl. Phys. Lett. 87 (2005) 113105.

[20] Y. Lee, E. Ahn, J. Kim, P. Moon, C. Yang, E. Yoon, H. Lim and H. Cheong, Complete suppression of large InAs island formation on GaAs by metalorganic chemical vapor deposition with periodic AsH3 interruption, Appl. Phys. Lett. 90 (2007) 033105.

[21] J. Tatebayashi, Y. Arakawa, N. Hatori, H. Ebe, M. Sugawara, H. Sudo and A. Kuramata, InAs/GaAs self assembled quantum dot lasers grown by metalorganic chemical vapor deposition - Effects of post-growth annealing on stacked InAs quantum dots, Appl. Phys. Lett. 85 (2004) 1024.

[22] Z. Yin, X. Tang, J. Zhao and S. Deny, Effects of growth conditions on InAs quantum dot formation by metalorganic chemical vapor deposition using tertiarybutylarsine in pure $\mathrm{N}_{2}$ ambient, Journal of Appl. Phys. 99 (2006) 124306.

[23] J. Tatebayashi, N. Hatori, M. Ishida, H. Ebe, M. Sugawara, Y. Arakawa, H. Sudo and A. Kuramata, $1.28 \mu \mathrm{m}$ lasing from stacked InAs/GaAs quantum dots with low temperature grown AlGaAs cladding layer by metalorganic chemical va por deposition, Appl. Phys. Lett. 86 (2005) 053107.

[24] Z. R. Wasilewski, S. Fafard, J. P. McCaffrey, Size and shape engineering of vertically stacked self-assembled quantum dots, J. Crystal Growth 201/202 (2008) 1131 .

[25] G. D. Lian, J. Yuan, L. M. Brown, G. H. Kim and D. A. Ritchie, Modification of InAs quantum dot structure by the growth of the capping layer, Appl. Phys. let. 73 (1998) 49.

[26] C. Paranthoen, N. Bertru, O. Dehaese, A. Le Corre, S. Loualiche, B. Lambert and G.

Patriarche, Height dispersion control of InAs/InP quantum dots emitting at $1.55 \mu \mathrm{m}$, Appl. Phys. Lett. 78 (2001) 1751. 
[27] Q. Xie, A. Madhukar, P. Shen and N. Kobayashi, Vertically self-organized InAs quantum box islands on GaAs(100), Phys. Rev. Lett. 75 (1995) 2542.

[28] J. Ruiz, Optical and morphological characterization of InAs quantum dots, Masters thesis, PUC-Rio, Brazil, 2015 (unpublished); and J. Ruiz, L. Dornelas, P. L. Souza, M. P. Pires, 2015 SBPMat Annual Meeting, Rio de Janeiro.

[29] R. B. Bergmann, A. Bill, On the origin of logarithmic-normal distributions: An analytical derivation and its application to nucleationand growth processes, J. Crystal Growth 310 (2008) 3135.

[30] E. Steimetz, J.-T.Zettler, F. Schienle, T. Trepk, T. Wethkamp, W. Richter,I. Sieber, In situ monitoring of InAs-on-GaAs quantum dot formation in MOVPE by reflectance-anisotropyspectroscopy and ellipsometry, Appl. Surf. Sci. 107 (1996) 203.

[31] E. Steimetz, T. Wehnert, H. Kirmse, F. Poser, J.-T.Zettler, W. Neumann, W. Richter, Optimizing the growth procedure for InAs quantum dot stacks by optical in situ techniques, J. Crystal Growth 221 (2000) 592.

[32] C. Weisbuch, R. Dingle, A. C. Gossard and W. Wiegmann, Optical characterization of interface disorder in GaAs-GaAIAs multi-quantum well structures, Solid Stae Commun. 38 (1981) 709.

[33] J.-P. Reithmaier, R. Höger and H. Riechert, Experimental evidence for the transition from two- to three-dimensional behavior of excitons in quantum-well structures, Physical Review B 43 (1991) 4933.

[34] D. W. Kim, Y. A. Leem, S. D. Yoo, D. H. Woo, D. H. Lee and J. C. Woo, Measurement of the exciton binding energy in a narrow $\mathrm{GaAs}-\mathrm{Al}_{\mathrm{x}} \mathrm{Ga}_{1-\mathrm{x}} \mathrm{As}$ quantum well by photoluminescence excitation spectroscopy, Physical Review B 47 (1993) 2042.

[35] G. Bastard, E. E. Mendez, L. L. Chang and L. Esaki, Exciton binding energy in quantum wells, Physical Review B 26 (1982) 1974.

[36] F. likawa, P. Motisuke, J. A. Brum, M.A. Sacilotti, A.P. Roth, R. A. Masut, Thermally induced In/Ga interdiffusion in $\ln _{x} G_{1-x} A s / G a A s$ strained single quantum well grown by LPMOVPE, J. Crystal Growth 93 (1988) 336.

[37] S.-S. Li, J.-B. Xia, Z. L. Yuan, Z. Y. Xu, W. Ge, X. R. Wang, Y. Wang, J. Wang, and L. L. Chang, Effective-mass theory for InAs/GaAs strained coupled quantum dots, Phys. Rev. B 54 (1996) 11575. 
[38] P. G. Linares, A. Martí, E. Antolin, A. Luque, III-V compound semiconductor screening for implementing quantum dot intermediate band solar cells, J. Appl. Phys. 109 (2011) 014313.

\section{Figure Captions}

Fig. 1 Cross-section HRTEM image of the InAs wetting layer and QD resulting from $12.0 \mathrm{~s}$ deposition time. The image is along the $<110>$ projection. The wetting layer is two monolayers thick, determined from the intensity profile (shown on the right side of the image).

Fig. 2 AFM images of the surface of the InAs grown on GaAs for 8.4 (a), 9.2 (b), 10.8 (c) and 12.0 $s(d)$. Quantum dots are observed only after $8.4 \mathrm{~s}$ deposition time.

Fig. 3 Histograms for the 1 and 5 periods samples for which the InAs layers were deposited for 10.8 ( $a$ and $b$ ) and $12.0 \mathrm{~s}$ ( $\mathrm{c}$ and $\mathrm{d}$ ).

Fig. 4 HREM images of the five period samples for which the InAs layer was deposited for 8.4 (a), 9.6 (b) and $12.0 \mathrm{~s}$ (c).

Fig. 5 RAS signal for the GaAs surface prior to InAs deposition and for InAs layers deposited for $3.6,4.8,6.0,7.2$ and $8.4 \mathrm{~s}$.

Fig. $6 \mathrm{PL}$ spectra in the quantum well energy range for the five period samples with InAs layers grown for different lengths of time.

Fig. 7 Calculated lowest optical transition energy as a function of InAs QW thickness (vertical dash). The line is just a guide for the eyes. The experimental PL peak energies for the different samples are introduced in the graph with different symbols according to the inset.

Fig. 8 Photoluminescence spectra of the samples for which the InAs layer was deposited for 8.4 s, $9.6 \mathrm{~s}, 10.8 \mathrm{~s}$ and $12.0 \mathrm{~s}$. These are the samples in which quantum dots were nucleated.

Fig. 9 Photoluminescence peak energy for the emissions attributed to the QDs as a function of QD height for the samples with different InAs deposition times represented by various symbols, as in the inset. The solid and dashed lines are a guide for the eyes connecting the theoretical calculation data assuming QD radius of $5 \mathrm{~nm}$ (solid) and $6 \mathrm{~nm}$ (dashed). 


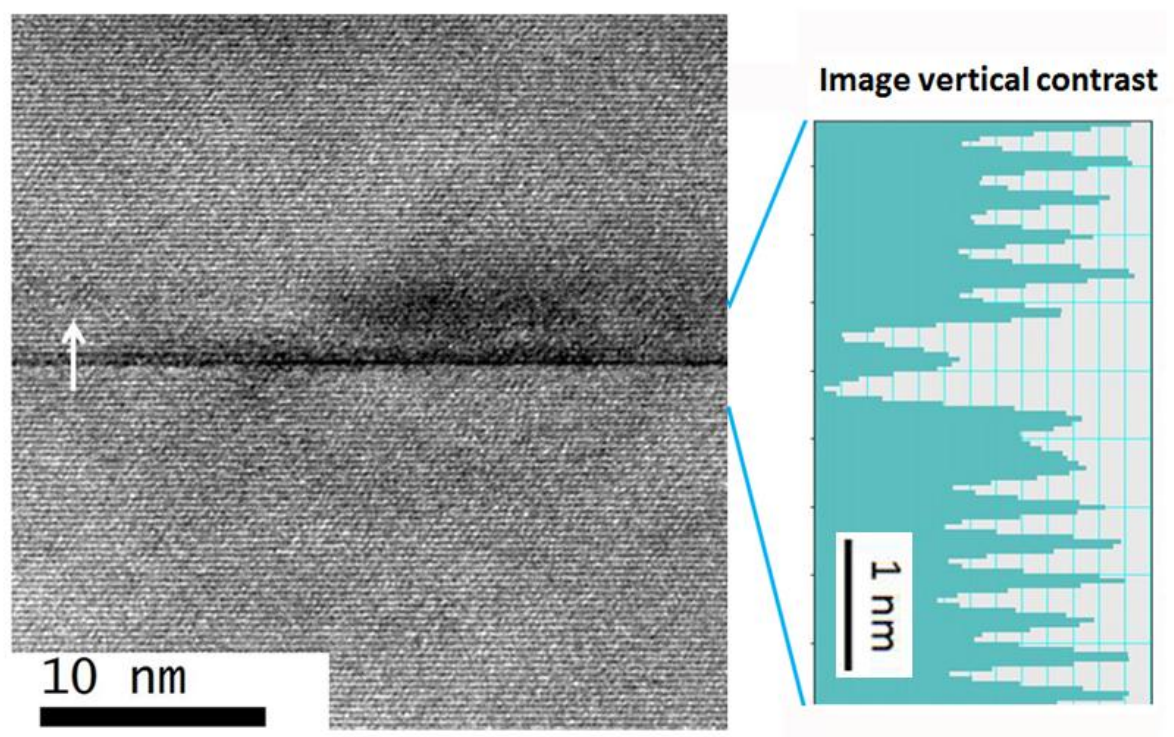

Fig. 1. Cross-section HRTEM image of the InAs wetting layer and QD resulting from $12.0 \mathrm{~s}$ deposition time. The image is along the $<110>$ projection. The wetting layer is two monolayers thick, as determined from the intensity profile (shown on the right side of the image).

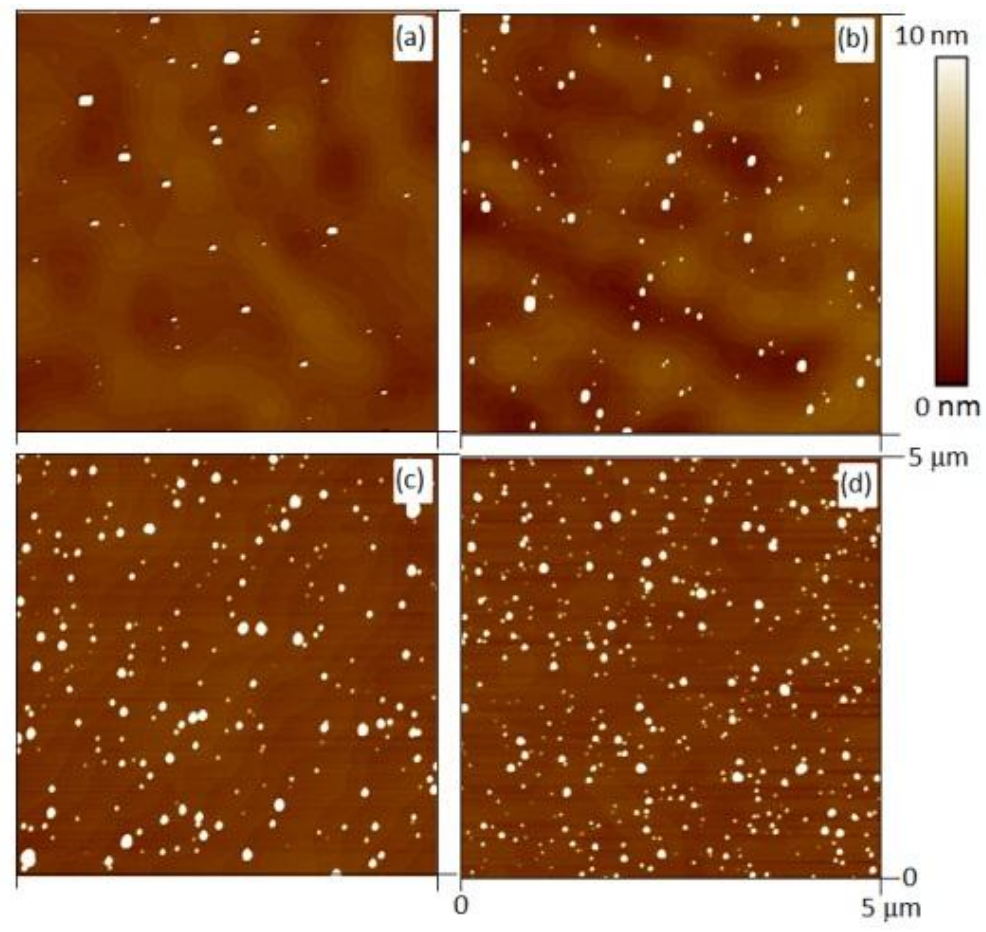

Fig. 2 AFM images of the surface of samples $5 \times 8.4$ (a), $5 \times 9.2$ (b), $5 \times 10.8$ (c) and $5 \times 12.0 \mathrm{~s}$ (d). Quantum dots are observed only after $8.4 \mathrm{~s}$ deposition time. 

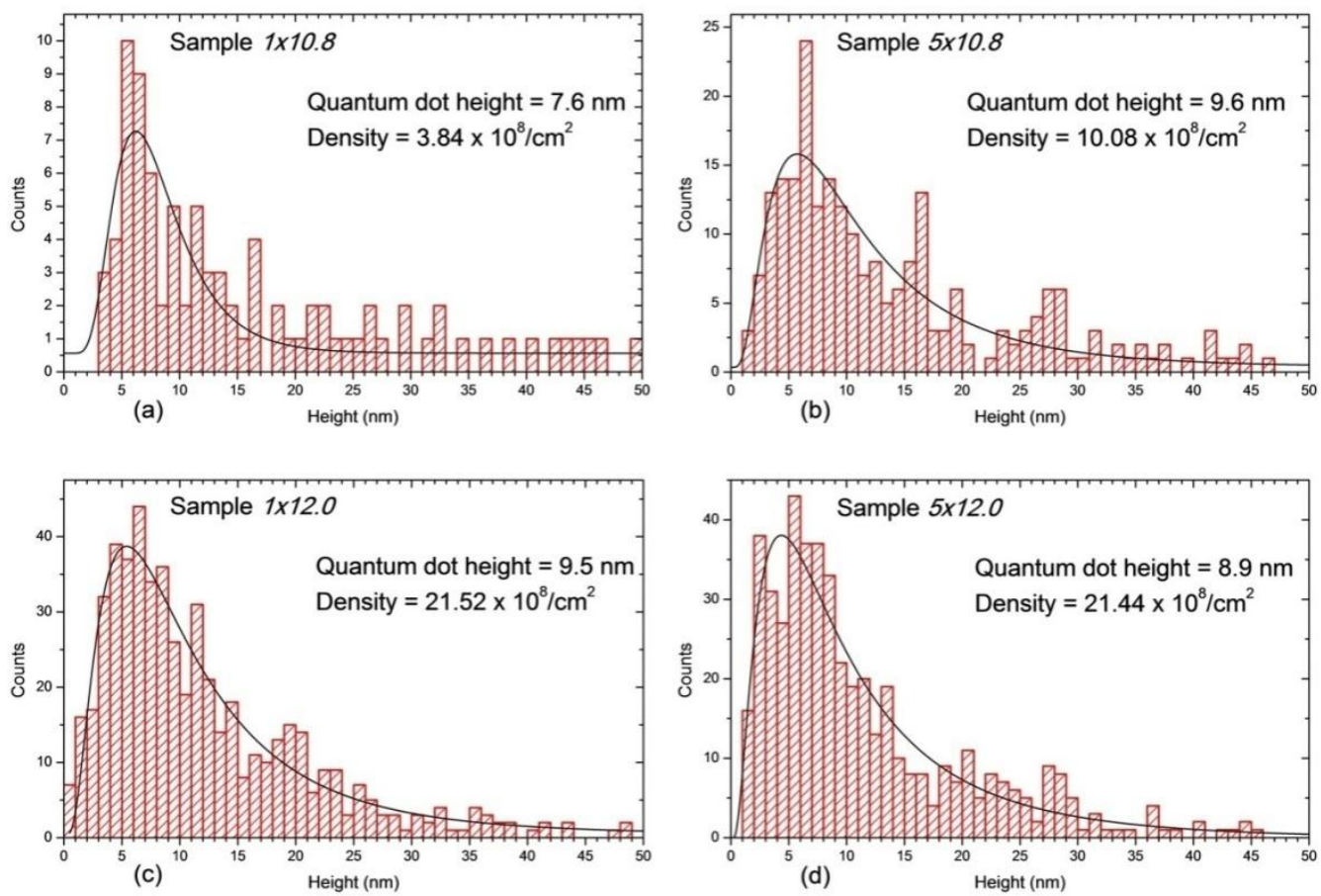

Fig. 3 Histograms for samples (a) $1 \times 10.8$, (b) $5 \times 10.8$, (c) $1 \times 12.0$ and (d) 5x12.0.

(a)

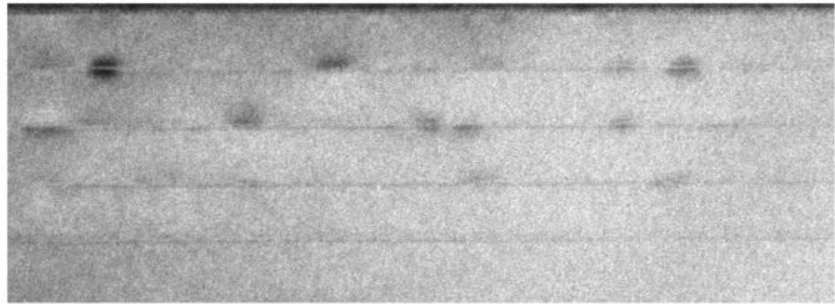

(b)

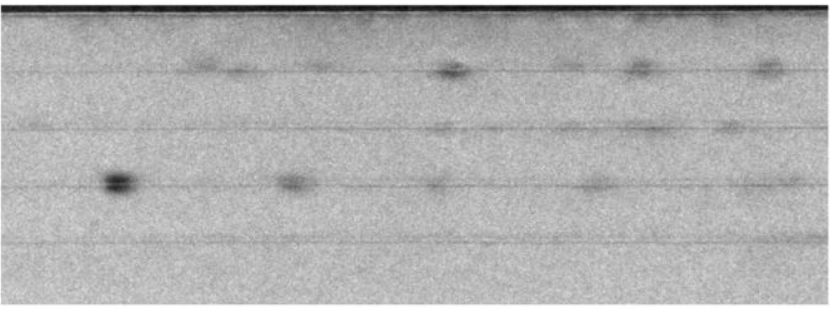

(c)

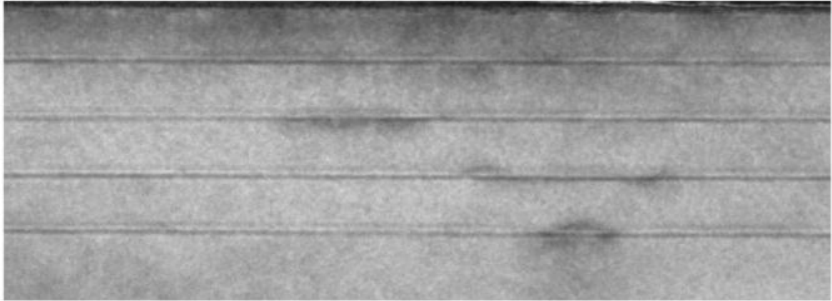

$100 \mathrm{~nm}$

Fig. 4 HREM images of samples (a) 5x8.4, (b) $5 \times 9.6$ and (c) $5 \times 12.0$. 


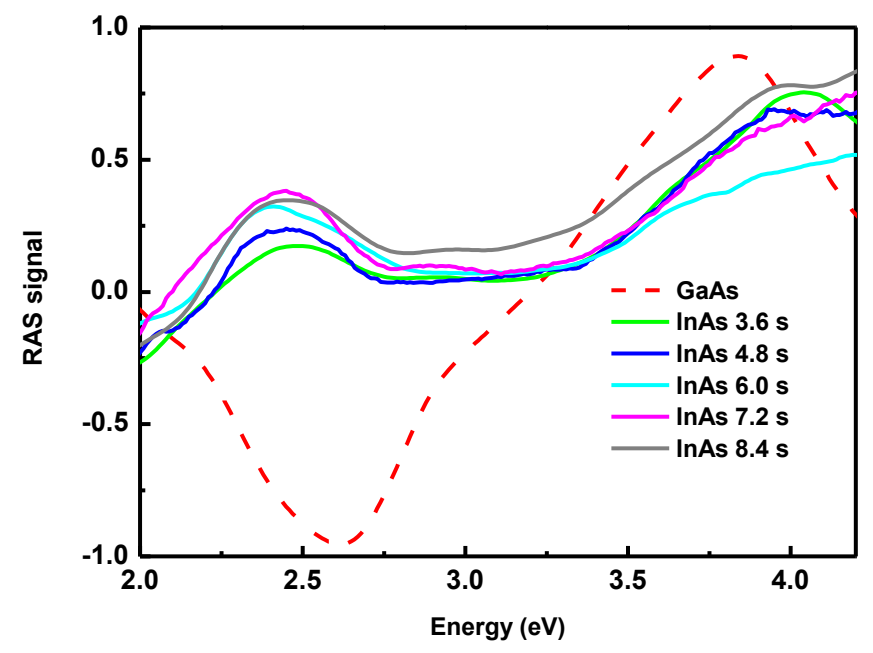

Fig. 5 RAS signal for the GaAs surface prior to InAs deposition and for InAs layers deposited for 3.6, 4.8, 6.0, 7.2 and $8.4 \mathrm{~s}$.

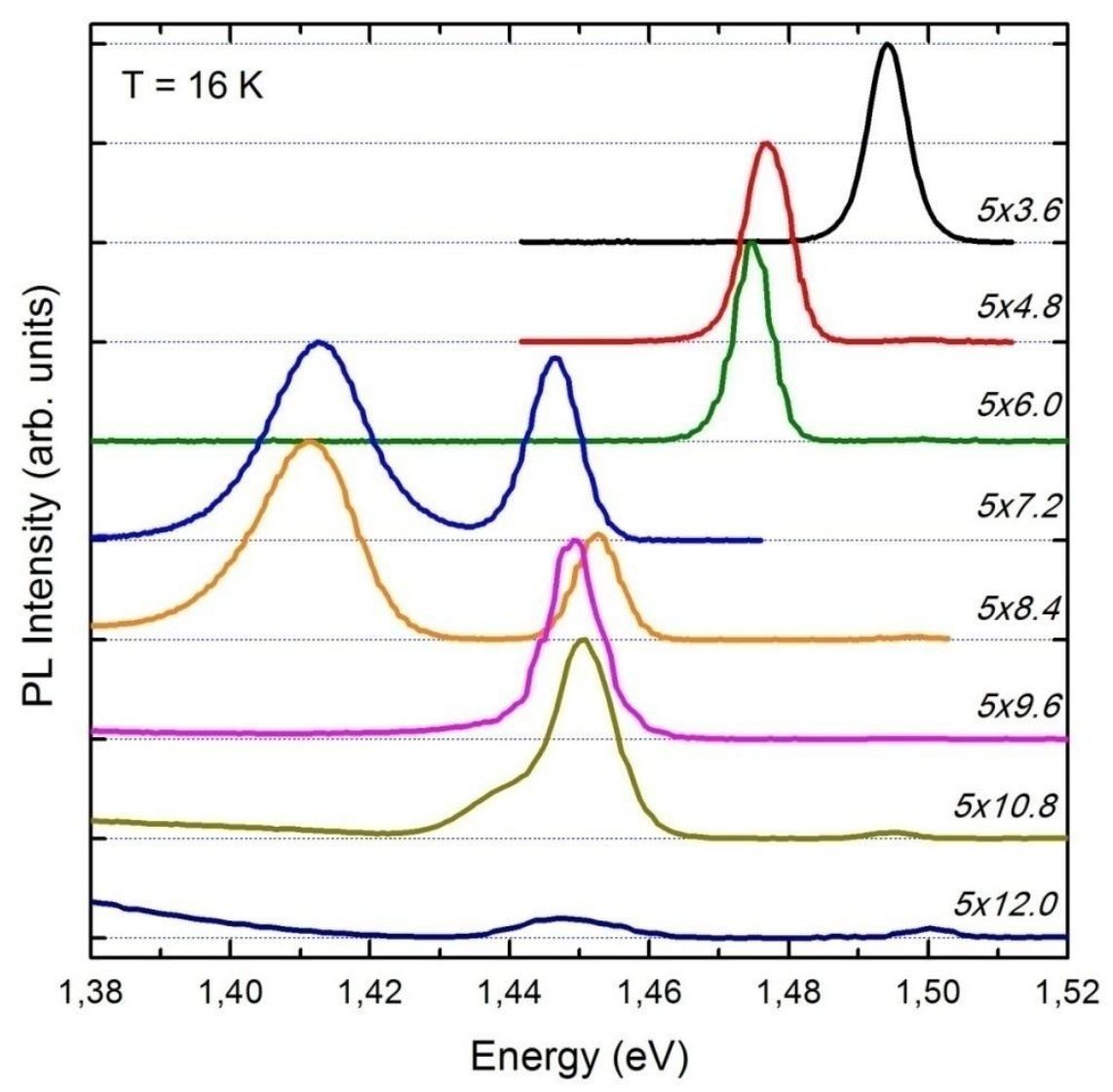

Fig. 6 PL spectra in the quantum well energy range for the five period samples with InAs layers grown for different lengths of time. 


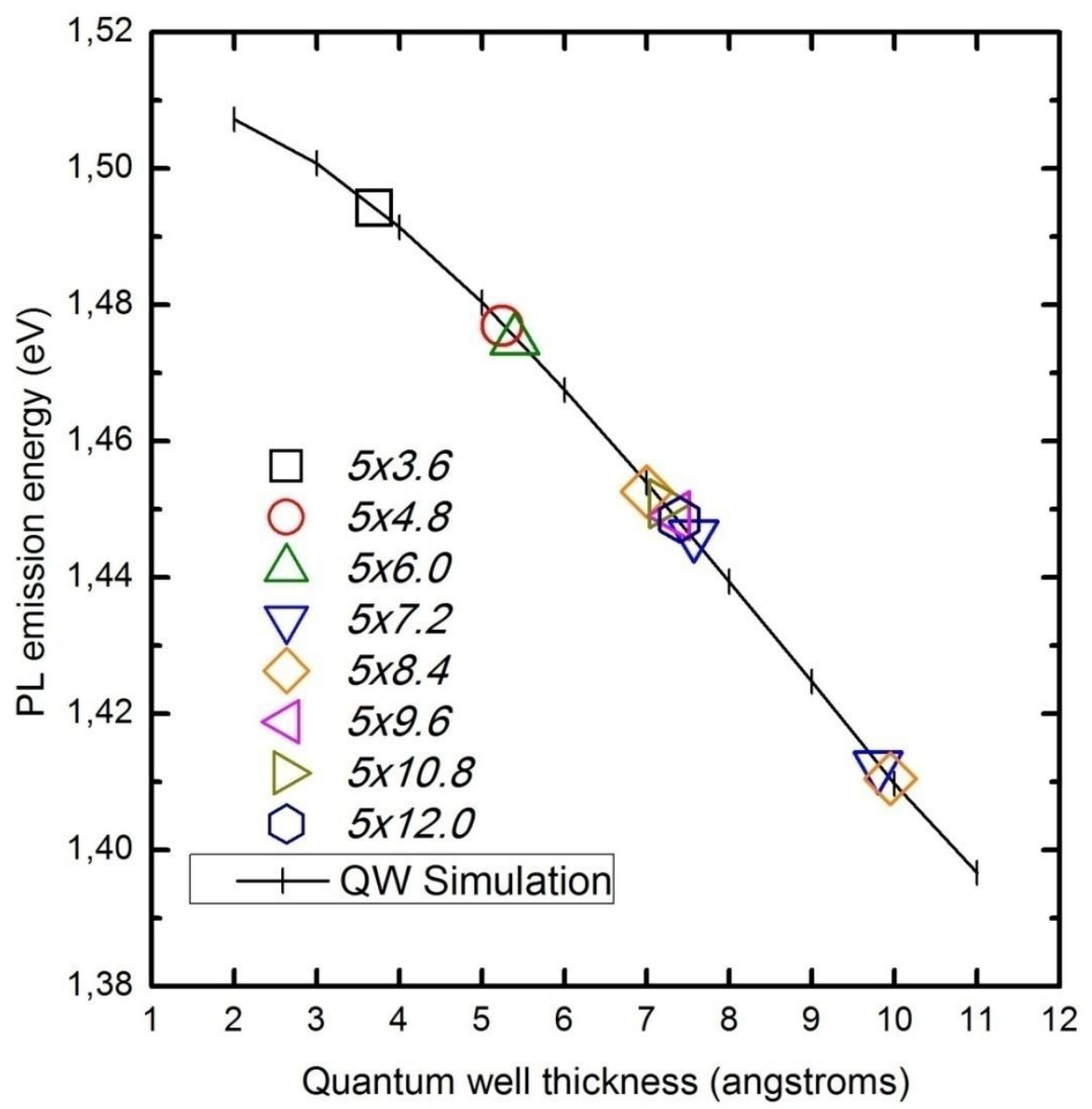

Fig. 7 Calculated lowest optical transition energy as a function of InAs QW thickness (vertical dash). The line is just a guide for the eyes. The experimental PL peak energies for the different samples are introduced in the graph with different symbols according to the inset. 


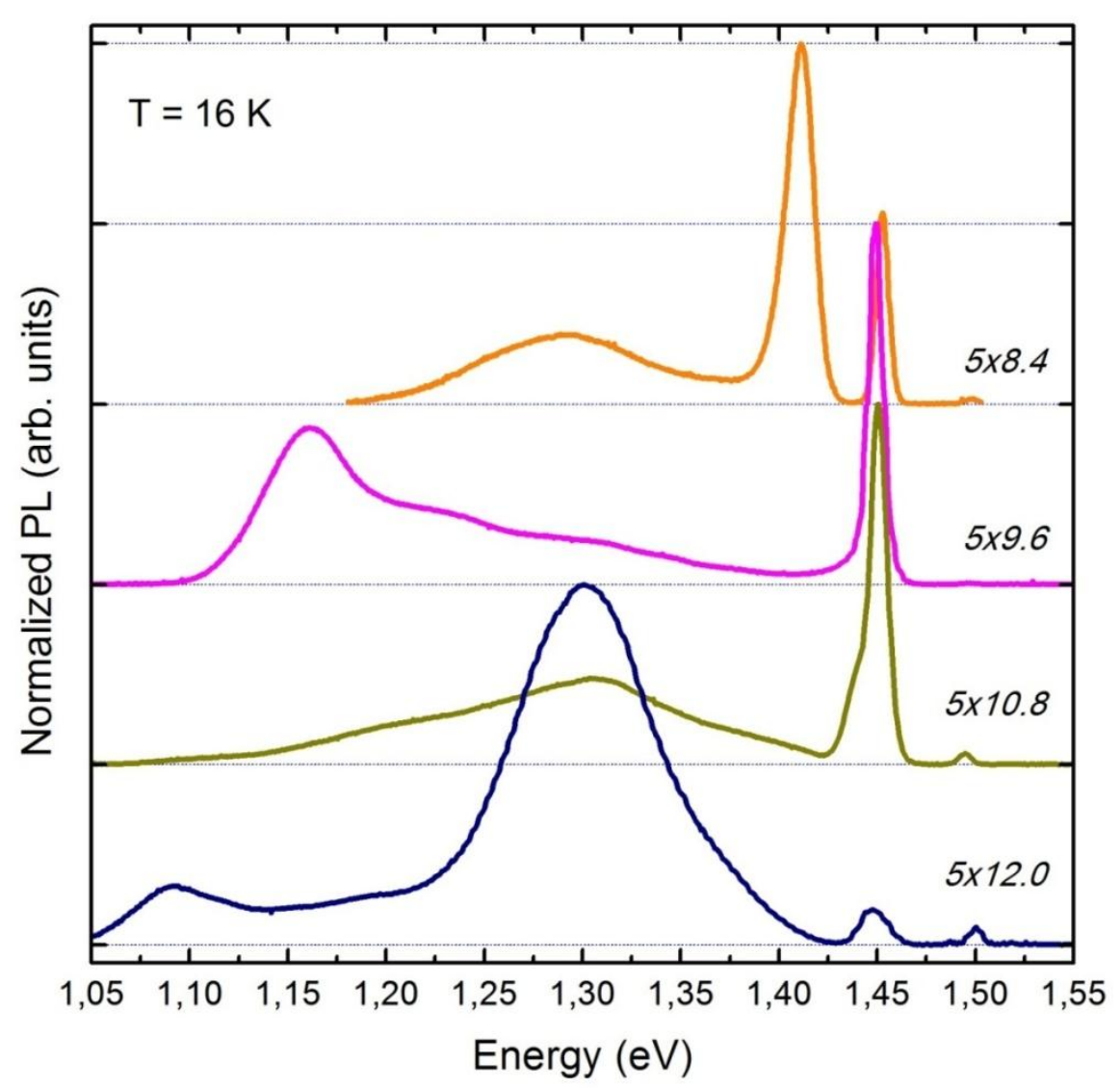

Fig. 8 Photoluminescence spectra of samples $5 \times 8.4,5 \times 9.6,5 \times 10.8$ and $5 \times 12.0$. These are the samples in which quantum dots were nucleated. 


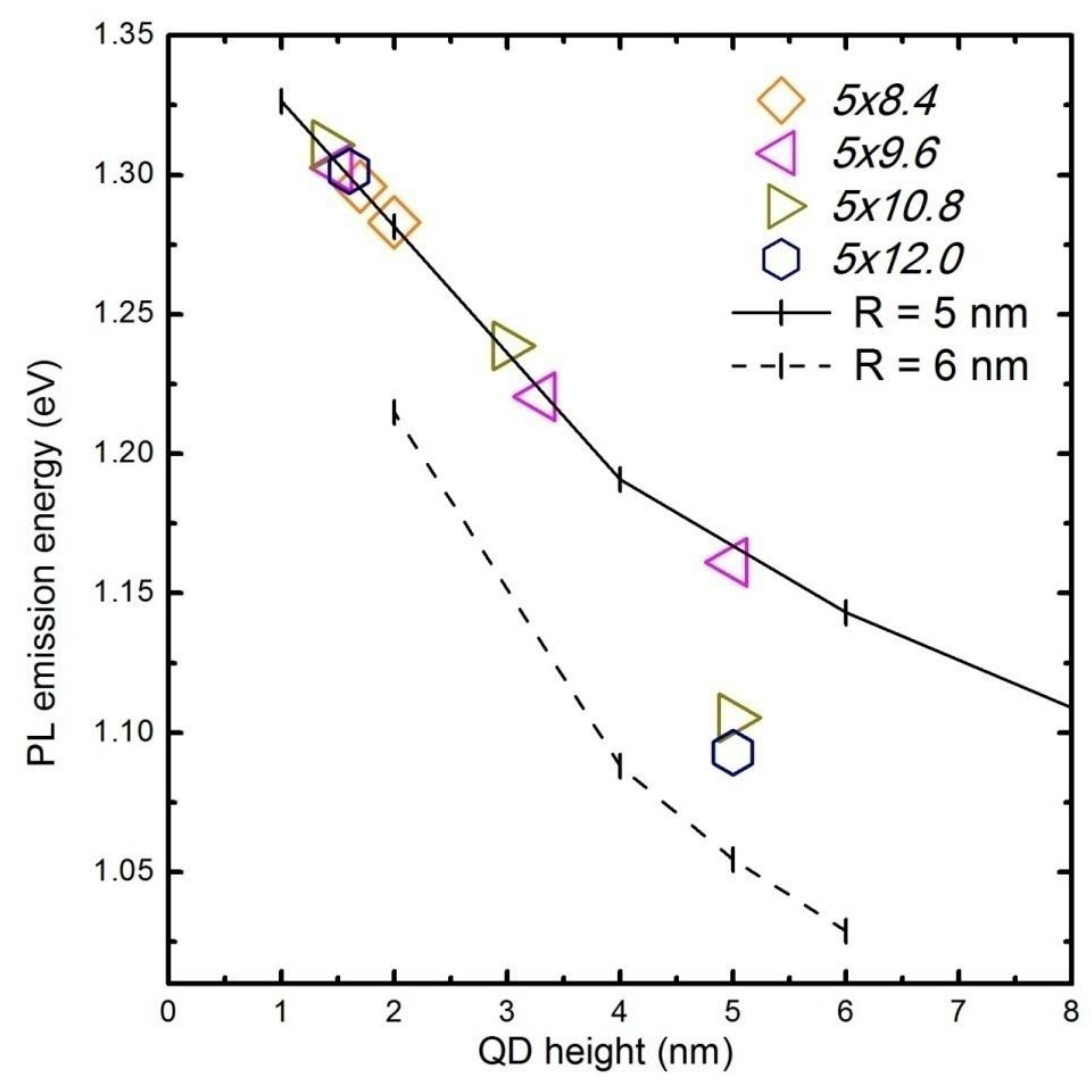

Fig. 9 Photoluminescence peak energy for the emissions attributed to the QDs as a function of $Q D$ height for the samples with different InAs deposition times represented by symbols. The solid and dashed lines are a guide for the eyes connecting the theoretical calculation data assuming $Q D$ radius of $5 \mathrm{~nm}$ (solid) and $6 \mathrm{~nm}$ (dashed). 